\title{
MACRONUTRIENTES NO SISTEMA SOLO-Pinus caribaea Morelet EM PLANTIOS APRESENTANDO AMARELECIMENTO DAS ACÍCULAS E MORTE DE PLANTAS ${ }^{1}$
}

\author{
Roselene de Queiroz Chaves² e Gilberto Fernandes Corrêa ${ }^{3}$
}

\begin{abstract}
RESUMO - Este estudo foi desenvolvido em áreas de Cerrado, no oeste de Minas Gerais, visando contribuir para o diagnóstico de causas que têm levado à morte precoce de pinheiros, verificada após amarelecimento e necrose progressiva das acículas. Foram comparadas árvores de Pinus caribaea Morelet das variedades caribaea e hondurensis, plantadas entre 1977-82. Em cada local de amostragem, coletou-se material de 20 árvores, sendo $10 \mathrm{com}$ amarelecimento das acículas e 10 normais. Foram coletadas acículas e tecidos do xilema e floema de raízes nos quais foram analisados os teores de N, P, K, Ca, Mg e S. A classe de solo dos sítios de estudo é Latossolo Amarelo ácrico típico. As amostras foram coletadas nas profundidades: 0-5, 5-10, 10-20, 2040, 40-60, 60-80, 80-100, 100-150, 150-200, 200-250 e 250-300 cm, nas quais se analisaram os teores trocáveis de $\mathrm{Ca}, \mathrm{Mg}, \mathrm{P}$ e $\mathrm{K}$, bem como o pH em água e em $\mathrm{KCl}$, a acidez extraível (H+Al) e o teor de matéria orgânica. Os teores de N, P, K e S não diferiram significativamente entre acículas verdes e amarelecidas, enquanto os teores de $\mathrm{Ca}$ e $\mathrm{Mg}$ foram significativamente inferiores nelas. Os sintomas visuais, confirmados pelos resultados analíticos, revelaram um quadro complexo de deficiências no sistema solo-planta estudado, envolvendo principalmente os macronutrientes $\mathrm{Ca}$ e $\mathrm{Mg}$.
\end{abstract}

Palavras-chave: Pinus tropical, relação solo-planta, deficiência nutricional, área de cerrado e reflorestamento.

MACRONUTRIENTS IN THE SOIL-Pinus caribaea Morelet SYSTEM WITH YELLOWING OF THE NEEDLES FOLLOWED BY SENESCENCE AND DEATH

\begin{abstract}
This study was carried out to diagnose senescence and pine trees death (Pinus caribaea Morelet), observed after yellowing and progressive necrosis of the needles in the Brazilian Cerrado in western Minas Gerais. The varieties caribaea and hondurensis, planted between 1977-82 were compared. In the three sites studied samples from twenty trees were collected; ten with yellowing of the needles and ten without symptoms. From each tree, phloem and xylem tissue from root segments and needles were collected and the contents of $\mathrm{N}, \mathrm{P}, \mathrm{K}, \mathrm{Ca}, \mathrm{Mg}$ and $\mathrm{S}$ were analyzed. The considered sites is formed by a Yellow Latosol (Typic Acrustox) with high clay content and the presence of positive charges (acric soil). Soil sampling was performed at the following depths: 0-5, 5-10, 10-20, 20-40, 40-60, 60-80, 80-100, 100-150, 150-200, 200-250 and 250$300 \mathrm{~cm}$. Soil analysis was carried out to evaluate the contents of organic matter, concentration of Ca, $\mathrm{Mg}$, $P$ and $K$ as well as $\mathrm{pH}$ in water and in $\mathrm{KCl}$. The mean concentration of $N, P, K$ and $S$ did not differ significantly in the green and yellow needles but Ca and Mg contents were significantly lower in the yellow needles. Visual symptoms and laboratory tests revealed complex deficiency symptoms involving Ca and $\mathrm{Mg}$.
\end{abstract}

Keywords: tropical pine, plant soil relations, nutritional deficiency, Brazilian Savanna (Cerrado), afforestation.

\footnotetext{
${ }^{1}$ Recebido em 07.08.2003 e aceito para publicação em 10.08.2005.

${ }^{2}$ Embrapa Arroz e Feijão, Caixa Postal 179, 75375-000 Santo Antônio de Goiás-Goiás. <roselene@ @npaf.embrapa.br>.

${ }^{3}$ Instituto de Ciências Agrárias (ICIAG) da Universidade Federal de Uberlândia (UFU), Caixa Postal 593, 38400-902 Uberlândia-MG.<gfcorrea@ufu.br>.
} 


\section{INTRODUÇÃO}

A silvicultura com espécies exóticas desempenha importante papel socioeconômico no Brasil, fornecendo matéria-prima para a produção de madeira, lenha, carvão e celulose, dentre outros. Todavia, essa atividade vem sendo conduzida sem a devida atenção para com a adequação das condições edáficas, mormente no que se aplica à correção das deficiências nutricionais do solo.

O impacto ambiental desta atividade tem sido questionado, com destaque no que se refere a alterações nas propriedades químicas e físicas das diferentes classes de solos utilizadas. Isso se deve, particularmente com o gênero Pinus, ao fato de que nutrientes minerais ficam retidos em quantidades consideráveis nos resíduos não decompostos das plantas, alterando o equilíbrio do sistema solo-planta.

É notório que a implantação de povoamentos florestais em áreas ecologicamente diferentes dos "habitats" de origem exige amplos conhecimentos técnicos para o sucesso do empreendimento, conforme observou Meneguzzi (1982).

A introdução de espécies exóticas no Brasil se deu, principalmente, com os gêneros Pinus (madeira de fibra longa) e Eucalyptus (madeira de fibra curta). Na maior parte, tais introduções foram bem-sucedidas, especialmente nas Regiões Sul e Sudeste, particularmente aquelas conduzidas por empresas que também investiram em pesquisas. Foi o que ocorreu com o gênero Eucalyptus, e os avanços aqui alcançados colocaram o Brasil em destaque no cenário internacional. Com o gênero Pinus, no entanto, isso não tem sido verificado.

Espécies tropicais, originárias da América Central, devido à sua rusticidade, tronco retilíneo propiciando apreciável rendimento volumétrico, mesmo em solos pobres e com déficit hídrico temporário, têm sido plantadas em áreas de cerrado, no Brasil central, sem o devido suporte da pesquisa.

Implantados principalmente no início da década de 1970, os maciços florestais de Pinus situam-se, em geral, nos chapadões extensamente dominados por latossolos com extrema pobreza nutricional. Há, portanto, limitações de ordem pedológica que, se não consideradas no manejo florestal, constituem ou induzem condições que podem estar na origem de problemas nutricionais que estariam levando os pinheiros a um processo de senescência precoce.

R. Árvore, Viçosa-MG, v.29, n.5, p.691-700, 2005
Há que se considerar, como fator agravante, o fato de se tratar de espécies exóticas perenes, cultivadas num espaçamento altamente competitivo. Estudos conduzidos na região de Iraí de Minas (MORAIS, 1999; SILVA, 2001) apontaram um histórico de manejo que atesta um descaso com a correção das deficiências do solo, tendo em vista a nutrição sustentável das plantas. Esse fato tem conduzido a um quadro de depauperamento e acidificação do solo. Em outra área, no Triângulo Mineiro, o processo de acidificação foi, também, observado por Lilienfein et al. (2000).

A morte precoce de pinheiros tem chamado a atenção de setores empresariais e da pesquisa, nessa região. A intensidade do problema varia com as condições edáficas, havendo certa relação entre as condições de drenagem do solo e a ocorrência dos sintomas de senescência de plantas.

Nos pinheiros com esse problema, o amarelecimento das acículas ocorre da ponta para a base, evoluindo para um tom pardo-avermelhado.

Esses sintomas de amarelecimento são mais intensos na parte superior da copa e se intensificam na planta, levando à queda das acículas, morte e posterior queda do pinheiro. Contudo, a ocorrência dessas sintomatologias não se dá em reboleiras, mas sim distribuída de forma aparentemente aleatória nos talhões, em árvores dominantes e dominadas.

Dada a importância da silvicultura no oeste de Minas Gerais, sobretudo com espécies de Pinus tropicais, devido ao diversificado parque industrial já implantado, é necessário um suporte da pesquisa, a fim de garantir a continuidade dessa atividade de forma sustentável. Objetivou-se com este estudo contribuir para o diagnóstico do quadro sintomático até então sem registros na literatura: morte precoce de pinheiros (Pinus caribaea Morelet), verificada após o amarelecimento e necrose progressiva das acículas.

\section{MATERIAL E MÉTODOS}

Os sítios de amostragem foram selecionados visando obter a máxima representatividade das condições de solo e dos sistemas silviculturais (preparo do solo, espécie, espaçamento, manejo florestal, idade) da maior parte dos reflorestamentos em áreas de cerrado do oeste de Minas Gerais. 
Foram escolhidos talhões de Pinus caribaea Morelet em idade comercial de corte, a fim de estudar áreas sob manejo florestal pelo maior tempo possível, apresentando pinheiros com sintomas bem característicos de senescência precoce (Figura 1).

Algumas características dos locais de estudo estão resumidas no Quadro 1. Procurou-se contemplar a classe de solo mais representativa das áreas regionalmente sob reflorestamento com Pinus, ou seja, o Latossolo Amarelo ácrico típico (EMBRAPA, 1999).

A amostragem do solo foi realizada a partir do preparo de uma seção de cortes (com o auxílio de enxadão e faca), que permitiu retirar as primeiras amostras (0-5, 5-10 e 10-20 cm). A seguir, nas demais profundidades (20-40, 40-60, 60-80, 80-100, 100-150, $150-200,200-250$ e $250-300 \mathrm{~cm}$ ), as amostragens foram feitas por tradagem. Assim, foram coletadas amostras simples em 11 seções até $300 \mathrm{~cm}$ de profundidade, compreendendo uma seção de cortes por sítio de estudo. Adotou-se, portanto, a sistemática empregada em levantamentos pedológicos durante a abertura de perfis para caracterização de uma classe de solo. A marcante isotropia do solo nos talhões amostrados foi determinante na adoção dessa sistemática de amostragem.


Figura 1 - Talhão de Pinus caribaea var. hondurensis apresentando plantas amarelecidas e outras já em franco processo de definhamento e morte (a); Planta de Pinus caribaea var. caribaea morta, ladeada por outras apresentando diferentes estádios/intensidades de amarelecimento/queda das acículas (b); Galhos coletados em plantas de Pinus caribaea var. caribaea normais (verde intenso) e anormais (amarelo) (c); e Aspecto das acículas de Pinus caribaea var. caribaea apresentando amarelecimento que progride da ponta para a base destas, evoluindo para uma coloração pardo-avermelhada (d).

Figure 1 -A Pinus caribaea var. hondurensis compartment showing yellowed plants and some of them already in a steady process of senescence and death (a); Dead Pinus caribaea var. caribaea plant, surrounded by plants showing different stages of the yellowing process and needle loss (b); Branches taken from healthy (intense green) and sick (yellow) Pinus caribaea var caribaea plants (c); Appearance of Pinus caribaea var. caribaea needles showing the yellowing increase from the tip to the basis of the needle, reaching a reddish-brown color $(d)$. 
Quadro 1 - Resumo das principais características descritivas dos locais I, II e III Table 1 -Summary of the main features of the sites I, II and III

\begin{tabular}{lccc}
\hline VARIÁVEIS & Local I & Local II & Local III \\
\hline Município & Uberlândia & Uberlândia & Romaria \\
Empresa & Pinusplan & Pinusplan & Satipel \\
Local & Floresta do Lobo & Floresta do Lobo & Nova Monte Carmelo S.A. \\
$\mathrm{N}^{0}$ do talhão & 18 & 302 & 1267 \\
Variedade & caribaea & caribaea & hondurensis \\
Ano do plantio & 1982 & 1978 & 1977 \\
Espaçamento $(\mathrm{m})$ & $2,50 \mathrm{x} 2,80$ & $2,00 \mathrm{x} 2,80$ & $2,00 \mathrm{x} 3,00$ \\
$\mathrm{~N}^{0}$ plantas/ha & 1428 & 1785 & 1667 \\
Adubação & $10 \mathrm{~g}$ supersimples/cova & 10 g supersimples/cova & Sem adubação \\
\hline
\end{tabular}

As amostras foram submetidas a análises químicas: $\mathrm{pH}_{\mathrm{H} 2 \mathrm{O}}$ e $\mathrm{pH}_{\mathrm{KCl}}, \mathrm{H}+\mathrm{Al}$, macronutrientes ( $\mathrm{P}, \mathrm{K}, \mathrm{Ca}$ e $\mathrm{Mg}$ ) e matéria orgânica, segundo Silva (1999). Essas análises foram realizadas no Laboratório de Análises de Solos (LABAS) do Instituto de Ciências Agrárias da Universidade Federal de Uberlândia (UFU).

A análise do material vegetal incluiu parte aérea (acículas) e de raízes (floema e xilema). Foram amostradas, em cada local, acículas de diferentes posições da copa, em 10 plantas normais (coloração verde-intenso) e em 10 plantas anormais (coloração amarela). Os tecidos do floema e do xilema foram coletados em raízes de quatro árvores normais e quatro anormais, em cada local. Esses materiais foram secados em estufa de ventilação forçada, a $65^{\circ} \mathrm{C}$, até peso constante, finamente moídos e submetidos às análises de $\mathrm{N}, \mathrm{P}, \mathrm{K}, \mathrm{Ca}, \mathrm{Mg}$ e S (SILVA, 1999). Essas análises foram realizadas no Laboratório de Solos Florestais do Departamento de Solos da Universidade Federal de Viçosa (UFV).

O tratamento estatístico empregado foi a estimação de parâmetros por intervalo, considerando-se que os pinheiros não foram amostrados ao acaso, já que no estudo proposto era necessário selecionar árvores com e sem sintomas de senescência precoce. Como as amostras eram pequenas, utilizou-se a distribuição t de "Student". Assim, a expressão utilizada para se calcular o intervalo de confiança da média foi:

$$
\left(\bar{x} \pm t_{n-1 ; \alpha / 2} s_{x} / \sqrt{n}\right)
$$

em que té o valor crítico da distribuição de Student à significância $\alpha=0,05$. Como o teste é bilateral, na expressão aparece: $\alpha / 2$. O fator $n$ refere-se ao número de árvores, e cada árvore corresponde a uma repetição. A interpretação a ser feita é a de que existe uma probabilidade de $95 \%$ do intervalo encontrado $(\chi)$ conter a verdadeira média da população. A hipótese $\mathrm{H}_{0}$ adotada foi a de que a concentração dos nutrientes no tecido vegetal é igual nos dois tipos de planta (normais e anormais). Assim, aceitou-se $\mathrm{H}_{0}$ quando os intervalos de confiança foram coincidentes entre plantas normais e anormais.

\section{RESULTADOS E DISCUSSÃO}

\subsection{Reação do solo e matéria orgânica}

Os dados apresentados no Quadro 2 indicam uma grande variação de $\Delta \mathrm{pH}$, da ordem de $-0,6 \mathrm{a}+1,7$, até 3,0 m de profundidade. Constatou-se, portanto, o caráter ácrico (EMBRAPA, 1999) bastante acentuado dos solos. Tal fato tem implicações particularmente importantes na nutrição de plantas. Significa que a capacidade de troca catiônica efetiva ( $\mathrm{t}$ ) desses solos é extremamente baixa (Quadro 2), encontrando-se essencialmente limitada à fração coloidal orgânica.

Nota-se, no Quadro 2, que nos locais amostrados, a partir de uma pequena profundidade, os valores de $\mathrm{pH}$ em $\mathrm{KCl}$ mostram-se superiores aos de $\mathrm{pH}$ em $\mathrm{H}_{2} \mathrm{O}$, resultando num $\Delta \mathrm{pH}$ positivo, o que evidencia uma inversão de carga. Tal efeito não ocorre nos primeiros centímetros do solo, devido às cargas negativas provenientes da matéria orgânica.

O caráter ácrico identificado no solo revela um predomínio de cargas positivas sobre as negativas, o que compromete a capacidade efetiva de troca de cátions. O histórico de uso dessas áreas com Pinus, corroborado pelos baixos valores de $\mathrm{pH}$ e pela virtual ausência de cálcio e magnésio (Quadros 2 e 7), revela que o plantio e a condução desses maciços florestais ocorreram sem que fosse realizado qualquer tipo de correção do solo, precedido apenas da retirada da vegetação original de cerrado. Nessa faixa de acidez,

R. Árvore, Viçosa-MG, v.29, n.5, p.691-700, 2005 
tratando-se de solos similares aos estudados por Corrêa (1989), também no oeste mineiro, os minerais da fração coloidal que dominam o complexo sortivo encontramse abaixo do ponto isoelétrico (OLIVEIRA, 2001). Isso equivale dizer que há um predomínio de cargas elétricas positivas, e nessa condição a CTC existente corresponde basicamente àquela relacionada a MO, conforme já mencionado.

Observa-se no Quadro 2 que, de acordo com a CFSEMG (1999), os teores de MO encontram-se em nível médio (2,1-4,0 dag. $\left.\mathrm{kg}^{-1}\right)$ até cerca de $100 \mathrm{~cm}$ de profundidade. Quando se analisam os valores da CTC efetiva, ou seja, aquela que o solo apresenta em sua condição natural, verifica-se uma estreita relação entre as variações desta com os teores de MO.

Apesar de produzir uma quantidade significativa de liteira, trabalhos têm mostrado que o teor de MO não é maior sob reflorestamento, quando este é comparado à vegetação natural, em áreas de cerrado (LILIENFEIN et al., 2000; LEPSCH, 1980; SOUZA e SOUZA, 1981). Essa informação requer maiores considerações, posto que a liteira do Pinus não representa um incremento efetivo de MO no solo, devido à demorada decomposição das acículas. Isso quer dizer que, por vários anos, a MO do solo relaciona-se àquela das condições anteriores ao plantio de Pinus. Ademais, a espessa liteira formada constitui uma proteção eficaz contra as oscilações térmicas e de umidade, assim tamponando as condições edafoclimáticas no nível do horizonte A. Essas condições estariam, por sua vez, favorecendo a biota do solo no processo de mineralização da MO residual (anterior ao reflorestamento), diminuindoa gradativamente. Essas considerações encontram respaldo na literatura (ZINN, 1998; MORAIS, 1999; SILVA, 2001).

Quadro 2 - Valores de $\mathrm{pH}$ e $\Delta \mathrm{pH}^{*}$ do solo, confrontados com os teores de matéria orgânica e CTC efetiva (t), nos locais I, II e III, até $300 \mathrm{~cm}$ de profundidade

Table 2 - Soil pH and $\triangle p H$ values against organic matter (MO) and CIC content in sites I, II and III up to $300 \mathrm{~cm}$ deep

\begin{tabular}{|c|c|c|c|c|c|c|c|c|c|c|}
\hline \multirow{2}{*}{$\begin{array}{l}\text { Prof. } \\
(\mathrm{cm})\end{array}$} & \multicolumn{5}{|c|}{ Local I } & \multicolumn{5}{|c|}{ Local II } \\
\hline & $\begin{array}{c}\mathrm{pH} \\
\mathrm{H}_{2} \mathrm{O}\end{array}$ & $\begin{array}{l}\mathrm{pH} \\
\mathrm{KCl}\end{array}$ & $\Delta \mathrm{pH}$ & $\begin{array}{c}\mathrm{MO} \\
\mathrm{dag} / \mathrm{kg}\end{array}$ & $\begin{array}{c}\mathrm{t} * * \\
\mathrm{cmol}_{\mathrm{c}} \cdot \mathrm{dm}^{-3}\end{array}$ & $\begin{array}{c}\mathrm{pH} \\
\mathrm{H}_{2} \mathrm{O}\end{array}$ & $\begin{array}{l}\mathrm{pH} \\
\mathrm{KCl}\end{array}$ & $\Delta \mathrm{pH}$ & $\begin{array}{c}\mathrm{MO} \\
\mathrm{dag} / \mathrm{kg}\end{array}$ & $\begin{array}{c}\mathrm{t} \\
\mathrm{cmol}_{\mathrm{c}} \cdot \mathrm{dm}^{-3}\end{array}$ \\
\hline $0-5$ & 4,8 & 4,2 & $-0,6$ & 3,9 & 1,01 & 4,7 & 4,2 & $-0,5$ & 3,7 & 1,32 \\
\hline $5-10$ & 4,8 & 4,4 & $-0,4$ & 3,6 & 0,91 & 4,8 & 4,5 & $-0,3$ & 3,1 & 0,81 \\
\hline $10-20$ & 4,9 & 4,5 & $-0,4$ & 3,1 & 0,90 & 4,8 & 4,7 & $-0,1$ & 2,8 & 0,40 \\
\hline $20-40$ & 4,9 & 4,7 & $-0,2$ & 3,1 & 0,60 & 4,8 & 4,8 & 0,0 & 2,7 & 0,30 \\
\hline $40-60$ & 5,0 & 4,9 & $-0,1$ & 2,6 & 0,66 & 4,6 & 5,0 & $+0,4$ & 2,6 & 0,25 \\
\hline $60-80$ & 5,0 & 5,1 & $+0,1$ & 2,3 & 0,35 & 4,4 & 5,2 & $+0,8$ & 2,4 & 0,15 \\
\hline $80-100$ & 5,1 & 5,5 & $+0,4$ & 2,0 & 0,25 & 4,5 & 5,5 & $+1,0$ & 2,1 & 0,15 \\
\hline $100-150$ & 5,2 & 5,8 & $+0,6$ & 1,8 & 0,15 & 4,7 & 6,1 & $+1,4$ & 1,7 & 0,15 \\
\hline $150-200$ & 5,2 & 6,2 & $+1,0$ & 1,4 & 0,15 & 5,3 & 6,5 & $+1,2$ & 1,5 & 0,05 \\
\hline $200-250$ & 5,4 & 6,5 & $+1,1$ & 1,2 & 0,06 & 5,5 & 6,7 & $+1,2$ & 1,1 & 0,06 \\
\hline $250-300$ & 5,5 & 6,6 & $+1,1$ & 1,1 & 0,05 & 5,7 & 6,8 & $+1,1$ & 1,1 & 0,06 \\
\hline \multirow{2}{*}{$\begin{array}{l}\text { Prof. } \\
(\mathrm{cm})\end{array}$} & \multicolumn{5}{|c|}{ Local III } & & & & & \\
\hline & $\begin{array}{c}\mathrm{pH} \\
\mathrm{H}_{2} \mathrm{O}\end{array}$ & $\begin{array}{l}\mathrm{pH} \\
\mathrm{KCl}\end{array}$ & $\Delta \mathrm{pH}$ & $\begin{array}{c}\text { MO } \\
\text { dag/kg }\end{array}$ & $\mathrm{cmol}_{\mathrm{c}}^{\mathrm{t}} \cdot \mathrm{dm}^{-3}$ & & & & & \\
\hline $0-5$ & 4,4 & 4,2 & $-0,2$ & 3,9 & 1,41 & & & & & \\
\hline $5-10$ & 4,3 & 4,3 & 0,0 & 3,5 & 1,30 & & & & & \\
\hline $10-20$ & 4,4 & 4,4 & 0,0 & 3,3 & 0,86 & & & & & \\
\hline $20-40$ & 4,6 & 4,6 & 0,0 & 3,0 & 0,57 & & & & & \\
\hline $40-60$ & 4,3 & 4,7 & $+0,4$ & 2,4 & 0,45 & & & & & \\
\hline $60-80$ & 4,4 & 5,0 & $+0,6$ & 2,1 & 0,25 & & & & & \\
\hline $80-100$ & 4,5 & 5,2 & $+0,7$ & 2,0 & 0,16 & & & & & \\
\hline $100-150$ & 4,4 & 5,4 & $+1,0$ & 1,9 & 0,15 & & & & & \\
\hline $150-200$ & 4,2 & 5,7 & $+1,5$ & 1,6 & 0,15 & & & & & \\
\hline $200-250$ & 4,3 & 6,0 & $+1,7$ & 1,4 & 0,15 & & & & & \\
\hline $250-300$ & 5,0 & 6,3 & $+1,3$ & 1,4 & 0,05 & & & & & \\
\hline
\end{tabular}

$* \Delta \mathrm{pH}=\mathrm{pH} \mathrm{KCl}-\mathrm{pH} \mathrm{H}_{2} \mathrm{O}$ e $* * \mathrm{t}=\mathrm{CTC}$ efetiva. 


\subsection{Macronutrientes no sistema solo-planta}

\section{Nitrogênio}

Apesar da estreita relação entre sintomas de amarelecimento e deficiência de nitrogênio, os teores encontrados para $\mathrm{N}$ nos três locais de amostragem não revelaram um quadro de deficiência desse nutriente nas plantas anormais, como pode ser observado no Quadro 3.

O Pinus apresenta baixa exigência em nitrogênio, apesar de ser este o macronutriente extraído em maior quantidade por este gênero de plantas (Van GOOR, 1966). Verifica-se, no Quadro 3, que houve diferença significativa quanto ao teor de $\mathrm{N}$ entre plantas normais e anormais somente na variedade hondurensis (Local III). Nessa variedade, as plantas amarelecidas apresentaram teores significativamente menores de $\mathrm{N}$ nas acículas, em relação às plantas normais.

Para o P. caribaea var. caribaea (Locais I e II), os dados indicam que o amarelecimento das acículas não se deve à deficiência de N. Todavia, no local III (variedade hondurensis) a deficiência de N pode estar contribuindo com o quadro de amarelecimento das acículas. A não-deficiência de $\mathrm{N}$ nos locais I e II deve estar relacionada com a menor exigência em $\mathrm{N}$ da variedade caribaea em relação à hondurensis. Apesar da pobreza nutricional do solo e do $\mathrm{N}$ seqüestrado na serapilheira, de difícil decomposição, é possível que a rizosfera de coníferas esteja mineralizando ou extraindo o $\mathrm{N}$ do solo que teria sido resistente à ação microbiana durante a vegetação anterior, conforme relataram Stone e Will (1965), citados por Lopes (1983).

Quadro 3 - Teores médios de $\mathrm{N}$ e intervalo de confiança ${ }^{1}$ no material vegetal de plantas normais e anormais de Pinus caribaea Morelet, nos três locais amostrados

Table 3 -Average $N$ content and confidence interval ${ }^{1}$ in the healthy and sick plant material of Pinus caribaea Morelet in the three sites sampled

\begin{tabular}{lcccc}
\hline Material & Pinheiros & \multicolumn{3}{c}{ Nitrogênio dag.kg Local II $^{-1}$} \\
\cline { 2 - 5 } Vegetal & & Local III & Local \\
\hline Acículas & Normais & $1,33 \pm 0,11$ & $1,12 \pm 0,13$ & $1,67 \pm 0,14^{2}$ \\
& Anormais & $1,30 \pm 0,09$ & $1,33 \pm 0,14$ & $1,40 \pm 0,12$ \\
Casca & Normais & $0,53 \pm 0,25$ & $0,64 \pm 0,19$ & $0,72 \pm 0,17$ \\
(floema) & Anormais & $0,45 \pm 0,23$ & $0,95 \pm 0,31$ & $1,02 \pm 0,49$ \\
Lenho & Normais & $0,16 \pm 0,02$ & $0,14 \pm 0,03$ & $0,12 \pm 0,01$ \\
Externo & Anormais & $0,17 \pm 0,02$ & $0,16 \pm 0,03$ & $0,13 \pm 0,02$ \\
(xilema) & & & & \\
\hline
\end{tabular}

${ }^{1}$ Intervalo de confiança obtido pelo teste t de Student a 5\% de significância ${ }^{2}$ diferença significativa, a significância de $5 \%$, pelo teste t de Student.

\section{Enxofre}

Poucos são os trabalhos que fazem referência a níveis de enxofre em Pinus. No Quadro 4, apresentamse os teores de $\mathrm{S}$ encontrados em Pinus caribaea neste estudo. A homogeneidade dos valores encontrados nos materiais vegetais analisados, nas duas variedades, nos três locais, revelam não ser o teor de S o responsável pelos sintomas de amarelecimento das acículas.

La Torraca et al. (1984) encontraram em $P$. elliottii, com 24 anos, respectivamente, 0,08; 0,07; e 0,05\% de $\mathrm{S}$ para acículas, casca e madeira. Segundo esses autores, a escala de retiradas totais de macronutrientes obedece à seguinte ordem: $\mathrm{N}>\mathrm{K}>\mathrm{Mg}>\mathrm{Ca}=\mathrm{S}>\mathrm{P}$, o que revela ser essa espécie pouco exigente deste nutriente. Valores da ordem de 0,09 e $0,15 \%$ de $\mathrm{S}$ em acículas de Pinus taeda foram encontrados por Vettorazzo e Couto (1997). Depreende-se, portanto, que o $P$. caribaea é menos exigente de $\mathrm{S}$ que outras espécies deste gênero.

\section{Fósforo e potássio}

Os valores encontrados nos macronutrientes fósforo e potássio no solo encontram-se no Quadro 5. Enquanto o K é o segundo macronutriente na escala de retiradas totais, o $\mathrm{P}$ é o último, sendo menos extraído que o S (LA TORRACA et al., 1984). Há certo consenso (BARROS e BRANDI, 1975; LOPES, 1983) de que o Pinus, por ser pouco exigente em P e K, não deve ser uma cultura causadora de desequilíbrios desses nutrientes no solo, no longo prazo.

Quadro 4 - Teores médios de S e intervalo de confiança ${ }^{1}$, no material vegetal de plantas normais e anormais de Pinus caribaea Morelet, nos três locais amostrados Table 4-Average $S$ content and confidence interval ${ }^{l}$ in the healthy and sick plant material of Pinus caribaea Morelet in the three sites sampled

\begin{tabular}{|c|c|c|c|c|}
\hline \multirow{2}{*}{$\begin{array}{l}\text { Material } \\
\text { Vegetal }\end{array}$} & \multirow[t]{2}{*}{ Pinheiros } & \multicolumn{3}{|c|}{ Enxofre dag. $\mathrm{kg}^{-1}(\%)^{2}$} \\
\hline & & Local I & Local II & Local III \\
\hline \multirow[t]{2}{*}{ Acículas } & Normais* & $0,018 \pm 0,001$ & $0,020 \pm 0,002$ & $0,024 \pm 0,004$ \\
\hline & Anormais & $0,017 \pm 0,001$ & $0,019 \pm 0,003$ & $0,023 \pm 0,002$ \\
\hline Casca & Normais & $0,013 \pm 0,004$ & $0,015 \pm 0,003$ & $0,012 \pm 0,003$ \\
\hline (floema) & Anormais & $0,017 \pm 0,004$ & $0,016 \pm 0,006$ & $0,013 \pm 0,001$ \\
\hline Lenho & Normais & $0,008 \pm 0,001$ & $0,009 \pm 0,002$ & $0,014 \pm 0,012$ \\
\hline $\begin{array}{l}\text { Externo } \\
\text { (xilema) }\end{array}$ & Anormais & $0,007 \pm 0,001$ & $0,007 \pm 0,001$ & $0,008 \pm 0,002$ \\
\hline
\end{tabular}

${ }^{1}$ Intervalo de confiança obtido pelo teste t de Student a $5 \%$ de significância ${ }^{2}$ Não houve diferença significativa, a 5\% de significância, pelo teste t de Student. 
Quadro 5 - Teores de P e K, até $3 \mathrm{~m}$ de profundidade, no solo dos três locais amostrados

Table $5-P$ and $K$ content, up to $3 m$ deep, in the soil of the three sites sampled

\begin{tabular}{ccccccc}
\hline \multirow{2}{*}{$\begin{array}{c}\text { Profundidade } \\
(\mathrm{cm})\end{array}$} & \multicolumn{4}{c}{ Fósforo e Potássio $\left(\mathrm{mg} . \mathrm{kg}^{-1}\right)$} \\
\cline { 2 - 7 } & \multicolumn{2}{c}{ Local I } & \multicolumn{2}{c}{ Local II } & \multicolumn{2}{c}{ Local III } \\
& $\mathrm{P}$ & $\mathrm{K}$ & $\mathrm{P}$ & $\mathrm{K}$ & $\mathrm{P}$ & $\mathrm{K}$ \\
\hline $0-5$ & 0,8 & 12,8 & 0,8 & 15,8 & 0,8 & 10,8 \\
$5-10$ & 0,5 & 11,8 & 0,6 & 9,8 & 0,5 & 8,8 \\
$10-20$ & 0,4 & 8,8 & 0,5 & 5,9 & 0,4 & 8,8 \\
$20-40$ & 0,4 & 7,8 & 0,5 & 4,9 & 0,4 & 9,8 \\
$40-60$ & 0,4 & 7,8 & 0,4 & 3,9 & 0,4 & 4,9 \\
$60-80$ & 0,6 & 4,9 & 0,7 & 2,9 & 0,7 & 3,9 \\
$80-100$ & 0,6 & 4,9 & 0,6 & 1,9 & 0,6 & 5,9 \\
$100-150$ & 0,5 & 3,9 & 0,4 & 2,9 & 0,5 & 4,9 \\
$150-200$ & 0,4 & 4,9 & 0,5 & 3,9 & 0,4 & 4,9 \\
$200-250$ & 0,5 & 5,9 & 0,5 & 3,9 & 0,4 & 2,9 \\
$250-300$ & 0,5 & 4,9 & 0,4 & 4,9 & 0,4 & 2,9 \\
\hline
\end{tabular}

Deve-se levar em conta também o fato de a liteira do Pinus ser muito resistente à decomposição, não disponibilizando, no curto prazo, parte do $\mathrm{P}$ ao sistema solo-planta. $\mathrm{O}$ mesmo raciocínio se aplica à dinâmica do potássio.

Lepsch (1980), comparando solos sob Pinus e cerrado nativo até $80 \mathrm{~cm}$ de profundidade, quanto a $\mathrm{P}$ e K, encontrou diferenças significativas somente para K, de 0-20 cm (menor teor sob Pinus). Zinn (1998) também notou uma redução do K disponível, somente na camada de $0-5 \mathrm{~cm}$ sob Pinus caribaea var. hondurensis, em comparação com o solo sob cerrado, até $60 \mathrm{~cm}$ de profundidade. Em relação ao $P$ não houve efeito significativo do plantio de Pinus. Lilienfein et al. (2000) não encontraram diferenças significativas quanto ao teor de K e P entre Pinus caribaea Morelet e cerrado nativo até $1,2 \mathrm{~m}$ de profundidade.

Os maiores teores de $\mathrm{K}$ nas camadas superficiais de solos sob cerrado, detectados por alguns autores (MORAIS, 1999; SILVA et al., 2001), podem estar relacionados às cinzas das freqüentes queimadas que ocorrem nessas áreas.

Os baixos valores encontrados no teor de $\mathrm{P}$ no solo, apesar de a literatura mostrar que não difere dos teores encontrados sob vegetação original de cerrado, podem guardar relação com os baixos valores de $\mathrm{pH}$, que controlam, de certa forma, a maneira pela qual o $\mathrm{P}$ é retido. Ou seja, dependendo do $\mathrm{pH}$, o sistema que envolve $\mathrm{Al}$ e $\mathrm{Fe}$ pode manter fixadas grandes proporções de fósforo total no solo.

No Quadro 6, apresentam-se os teores de P e K no material vegetal. Esses valores estão acima dos encontrados por La Torraca et al. (1984) para P. elliottii: 0,05; 0,03; e $0,02 \%$ de P e 0,22; 0,11 ; e $0,05 \%$ de K, respectivamente nas acículas, casca e madeira. Esses autores citaram os teores máximo e mínimo de $\mathrm{P}$ e K nas acículas, segundo Zöttl (1973), a saber: 0,13-0,20\% de $\mathrm{P}$ e $0,6-1,2 \%$ de $\mathrm{K}$, enquanto valores menores que $0,09 \%$ para $\mathrm{P}$ e $0,4 \%$ para $\mathrm{K}$ resultaram em crescimento deficiente.

Verificam-se, no Quadro 6, teores bem mais elevados de $\mathrm{K}$ em relação ao $\mathrm{P}$, o que concorda com a escala de extração de macronutrientes em Pinus, já apresentada.

Quadro 6 - Teores médios de P e K e intervalo de confiança ${ }^{1}$, no material vegetal de plantas normais e anormais de Pinus caribaea Morelet, nos três locais amostrados

Table 6 - Average $P$ and $K$ content and confidence interval ${ }^{1}$ in the healthy and sick plant material of Pinus caribaea Morelet in the three sites sampled

\begin{tabular}{|c|c|c|c|c|c|c|c|}
\hline \multirow{3}{*}{$\begin{array}{l}\text { Material } \\
\text { Vegetal }\end{array}$} & \multirow[t]{3}{*}{ Pinheiros } & \multicolumn{6}{|c|}{ Fósforo e Potássio dag. $\mathrm{kg}^{-1}(\%)^{2}$} \\
\hline & & \multicolumn{2}{|c|}{ Local I } & \multicolumn{2}{|c|}{ Local II } & \multicolumn{2}{|c|}{ Local III } \\
\hline & & $\mathrm{P}$ & $\mathrm{K}$ & $\mathrm{P}$ & $\mathrm{K}$ & $\mathrm{P}$ & $\mathrm{K}$ \\
\hline \multirow[t]{2}{*}{ Acículas } & Normais & $0,09 \pm 0,009$ & $0,45 \pm 0,072$ & $0,08 \pm 0,013$ & $0,39 \pm 0,090$ & $0,09 \pm 0,009$ & $0,38 \pm 0,112$ \\
\hline & Anormais & $0,12 \pm 0,018$ & $0,51 \pm 0,044$ & $0,09 \pm 0,008$ & $0,33 \pm 0,045$ & $0,11 \pm 0,027$ & $0,37 \pm 0,077$ \\
\hline Casca & Normais & $0,02 \pm 0,015$ & $0,30 \pm 0,295$ & $0,04 \pm 0,020$ & $0,13 \pm 0,127$ & $0,03 \pm 0,004$ & $0,11 \pm 0,013$ \\
\hline (floema) & Anormais & $0,01 \pm 0,006$ & $0,30 \pm 0,190$ & $0,05 \pm 0,029$ & $0,16 \pm 0,074$ & $0,02 \pm 0,001$ & $0,14 \pm 0,050$ \\
\hline Lenho & Normais & $0,01 \pm 0,005$ & $0,08 \pm 0,028$ & $0,01 \pm 0,003$ & $0,03 \pm 0,011$ & $0,01 \pm 0,000$ & $0,04 \pm 0,007$ \\
\hline $\begin{array}{l}\text { Externo } \\
\text { (xilema) }\end{array}$ & Anormais & $0,01 \pm 0,005$ & $0,12 \pm 0,049$ & $0,01 \pm 0,004$ & $0,05 \pm 0,002$ & $0,01 \pm 0,000$ & $0,04 \pm 0,009$ \\
\hline
\end{tabular}

${ }^{1}$ Intervalo de confiança obtido pelo teste t de Student a $5 \%$ de significância.

${ }^{2}$ Não houve diferença significativa, a $5 \%$ de significância pelo teste t de Student. 
Diante dos dados analíticos deste estudo e da contribuição de outros autores, pode-se dizer que as duas variedades de Pinus em discussão são bastante eficientes na utilização dos modestos teores de $\mathrm{P}$ e K do solo, já que os teores na planta estão, em geral, um pouco abaixo, ou no limite, dos níveis considerados como críticos pela literatura, enquanto no solo os teores são bem menores do que nos experimentos dos autores supracitados, que determinaram esses níveis críticos.

Vale ressaltar, todavia, que tais níveis críticos não se referem à espécie objeto deste estudo. Ademais, a análise estatística revelou que os teores de P e K encontrados nas acículas não estão relacionados com o amarelecimento destas, estando, possivelmente, acima do nível crítico para essas variedades de P. caribaea, em latossolos muito argilosos, fase cerrado.

\section{Cálcio e magnésio}

Verificou-se um quadro crítico com relação a Ca e Mg. Analisando os dados do Quadro 7, observase uma virtual ausência desses nutrientes no solo, nos três locais estudados. Níveis muito baixos desses dois macronutrientes eram de se esperar, em virtude do caráter ácrico dos solos.

Os dados apresentados no Quadro 7 indicam que a carência extrema de cálcio e magnésio se estende em profundidade, indicando prosseguir além dos 3,0 $\mathrm{m}$. No caso do $\mathrm{Mg}$, nada foi detectado desde a superfície do solo, enquanto os teores de $\mathrm{Ca}$, extremamente baixos na porção superior do solo, anulam-se com a profundidade.

Quadro 7 - Teores de $\mathrm{Ca}$ e $\mathrm{Mg}$, até $3 \mathrm{~m}$ de profundidade, no solo dos três locais amostrados

Table $7-C a$ and $M g$ content, up to $3 m$ deep, in the soil of the three sites sampled

\begin{tabular}{ccccccc}
\hline \multirow{2}{*}{$\begin{array}{c}\text { Profundidade } \\
(\mathrm{cm})\end{array}$} & \multicolumn{6}{c}{ Cálcio e Magnésio $\left(\mathrm{mg} . \mathrm{kg}^{-1}\right)$} \\
\cline { 2 - 7 } & \multicolumn{2}{c}{ Local I } & \multicolumn{2}{c}{ Local II } & \multicolumn{2}{c}{ Local III } \\
& $\mathrm{Ca}$ & $\mathrm{Mg}$ & $\mathrm{Ca}$ & $\mathrm{Mg}$ & $\mathrm{Ca}$ & $\mathrm{Mg}$ \\
\hline $0-5$ & 0,1 & 0,0 & 0,1 & 0,0 & 0,1 & 0,0 \\
$5-10$ & 0,1 & 0,0 & 0,1 & 0,0 & 0,1 & 0,0 \\
$10-20$ & 0,1 & 0,0 & 0,1 & 0,0 & 0,1 & 0,0 \\
$20-40$ & 0,1 & 0,0 & 0,1 & 0,0 & 0,1 & 0,0 \\
$40-60$ & 0,1 & 0,0 & 0,1 & 0,0 & 0,1 & 0,0 \\
$60-80$ & 0,1 & 0,0 & 0,1 & 0,0 & 0,1 & 0,0 \\
$80-100$ & 0,0 & 0,0 & 0,0 & 0,0 & 0,1 & 0,0 \\
$100-150$ & 0,0 & 0,0 & 0,0 & 0,0 & 0,0 & 0,0 \\
$150-200$ & 0,0 & 0,0 & 0,0 & 0,0 & 0,0 & 0,0 \\
$200-250$ & 0,0 & 0,0 & 0,0 & 0,0 & 0,0 & 0,0 \\
$250-300$ & 0,0 & 0,0 & 0,0 & 0,0 & 0,0 & 0,0 \\
\hline
\end{tabular}

Esse cenário revela que, por menos exigente que o P. caribaea seja com relação a esses nutrientes, certamente apresentará problemas nutricionais decorrentes da escassez de $\mathrm{Ca}$ e $\mathrm{Mg}$ nesses pedoambientes. Tais problemas têm como fator agravante a lenta decomposição da liteira, o que representa uma ruptura no processo de ciclagem de nutrientes.

Por menor que seja a quantidade de Ca e Mg imobilizada na liteira, é de se esperar que, em se tratando de um solo profundamente dessaturado, esta reserva teria papel relevante na nutrição dos pinheiros nesse sistema.

Estudo realizado por Chijioke (1980) chamou a atenção para o acúmulo de $\mathrm{Ca}$ e $\mathrm{Mg}$ na liteira de Pinus caribaea em solos pouco férteis do Brasil, o que evidencia uma imobilização desses elementos pela liteira, à medida que esta se mostra resistente à decomposição. Em solos sob vegetação nativa de cerrado, Lepsch (1980) encontrou teores significativamente menores de $\mathrm{Ca}$ e $\mathrm{Mg}$ sob Pinus do que sob cerrado, na camada de $0-20 \mathrm{~cm}$.

Os teores de $\mathrm{Ca}$ e $\mathrm{Mg}$ encontrados no material vegetal revelaram que os baixos teores desses nutrientes no solo estão acarretando um visível quadro de deficiência nutricional nas plantas.

No Quadro 8, apresentam-se os teores de Ca e $\mathrm{Mg}$ encontrados no material vegetal. Observa-se uma nítida deficiência, revelada pelos teores significativamente maiores desses nutrientes nas plantas normais, em relação àquelas amarelecidas (anormais).

A análise desses dados revela que existe cerca de sete vezes mais Ca e três vezes mais $\mathrm{Mg}$ nas acículas normais do que nas anormais. $\mathrm{O}$ fato de a diferença significativa ter ocorrido apenas em acículas é compreensível, dada a grande ação desses macronutrientes no aparato fotossintético. O Mg é, afinal, o átomo central da molécula de clorofila.

Em relação aos níveis críticos, Zöttl (1973), citado por Vettorazzo e Couto (1997), relatou que teores menores que 0,05 dag. $\mathrm{kg}^{-1}(\%)$ nas acículas, tanto para Ca quanto para $\mathrm{Mg}$, podem ocasionar um crescimento deficiente do Pinus.

Tomando como referência os autores supracitados, verifica-se que os teores de $\mathrm{Ca}$ e $\mathrm{Mg}$ encontrados nas acículas amarelecidas estavam abaixo dos níveis considerados como críticos. Os sintomas visuais confirmam esses resultados, já que coincidem com o que foi descrito por Malavolta (1974), como sintomas de deficiência de Ca e Mg em Pinus. 
Quadro 8 - Teores médios de Ca e Mg e intervalo de confiança ${ }^{1}$, no material vegetal de plantas normais e anormais de Pinus caribaea Morelet, nos três locais amostrados

Table 8 -Average Ca and Mg content and confidence interval ${ }^{1}$ in the healthy and sick plant material of Pinus caribaea Morelet in the three sites sampled

\begin{tabular}{lccccccr}
\hline Material & Pinheiros & \multicolumn{6}{c}{ Fósforo e Potássio dag.kg-1 $(\%)^{2}$} \\
\cline { 3 - 8 } & & \multicolumn{2}{c}{ Local I } & \multicolumn{2}{c}{ Local II } & \multicolumn{2}{c}{ Local III } \\
\cline { 3 - 8 } & & $\mathrm{Ca}$ & $\mathrm{Mg}$ & $\mathrm{Ca}$ & $\mathrm{Mg}$ & $\mathrm{Ca}$ & $\mathrm{Mg}$ \\
\hline Acículas & Normais & $0,10 \pm 0,016$ & $0,04 \pm 0,005$ & $0,20 \pm 0,036$ & $0,04 \pm 0,008$ & $0,06 \pm 0,013$ & $0,03 \pm 0,004$ \\
& Anormais & $0,02 \pm 0,007$ & $0,01 \pm 0,002$ & $0,02 \pm 0,009$ & $0,01 \pm 0,001$ & $0,01 \pm 0,005$ & $0,01 \pm 0,001$ \\
Casca & Normais & $0,03 \pm 0,021$ & $0,01 \pm 0,005$ & $0,10 \pm 0,062$ & $0,02 \pm 0,005$ & $0,09 \pm 0,044$ & $0,02 \pm 0,003$ \\
(floema) & Anormais & $0,04 \pm 0,031$ & $0,01 \pm 0,006$ & $0,10 \pm 0,027$ & $0,02 \pm 0,013$ & $0,08 \pm 0,035$ & $0,02 \pm 0,005$ \\
Lenho & Normais & $0,01 \pm 0,007$ & $0,01 \pm 0,002$ & $0,02 \pm 0,014$ & $0,01 \pm 0,002$ & $0,01 \pm 0,009$ & $0,01 \pm 0,001$ \\
Externo & Anormais & $0,01 \pm 0,001$ & $0,01 \pm 0,002$ & $0,01 \pm 0,009$ & $0,01 \pm 0,002$ & $0,01 \pm 0,010$ & $0,01 \pm 0,002$ \\
(xilema) & & & & & & &
\end{tabular}

${ }^{1}$ Intervalo de confiança obtido pelo teste t de Student a $5 \%$ de significância.

${ }^{2}$ Houve diferença significativa a $5 \%$ de significância, pelo teste $\mathrm{t}$ de Student.

O fato de num talhão de uma mesma variedade existirem árvores normais e anormais lado a lado reforça a hipótese da variabilidade genética das sementes utilizadas na formação das mudas que deram origem aos maciços florestais em estudo. Essa variabilidade genética reflete o padrão de comportamento de cada pinheiro, no que tange à eficiência na absorção e utilização de nutrientes. A heterogeneidade das plantas, tanto no que se refere ao desenvolvimento quanto à manifestação ou não de amarelecimento, bem como o fato de as sementes procederem de coleta em florestas nativas ${ }^{1}$, convergem em favor dessa hipótese. Ademais, não se trata de uma heterogeneidade relativa ao solo.

\section{CONCLUSÃO}

Os sintomas visuais de amarelecimento das acículas, culminando num marcante processo de senescência e morte de pinheiros, juntamente com os resultados analíticos do solo e do material vegetal, revelam um quadro complexo de deficiências no sistema solo-planta estudado, envolvendo principalmente os elementos cálcio e magnésio.

A acentuada deficiência de nutrientes no solo, agravada por um espaçamento que impõe grande competição entre plantas, predispõe o desenvolvimento do processo de amarelecimento das acículas, seguido de definhamento e morte de pinheiros. Esse quadro evidencia uma origem primária de natureza genética, enquanto a causa determinante se relaciona a fatores do meio, essencialmente a deficiência de nutrientes, agravada pela competição entre plantas.

A implantação e condução desses maciços florestais sem a correção das limitações nutricionais do solo foram a causa do problema. Este se agravou pela natural resistência das acículas à decomposição, rompendo a reciclagem dos nutrientes. A natureza nutricional do problema indica que ele pode ser evitado em novos plantios, mediante a devida correção das deficiências do solo e adequado manejo silvicultural.

\section{AGRADECIMENTOS}

À Satipel Florestal e sua equipe técnica e à Pinusplan Reflorestadora Ltda., pelo apoio à realização deste trabalho; à UFV, na pessoa do Prof. Dr. Nairam Félix de Barros, onde parte das análises foram realizadas; e à CAPES, pela bolsa de estudo.

\section{REFERÊNCIAS BIBLIOGRÁFICAS}

BARROS, N.F.; BRANDI, R.M. influência de três espécies florestais sobre a fertilidade de solo de pastagem em Viçosa, M.G. Brasil Florestal, n. 6 , v. 21 , p. 24-29, 1975.

\footnotetext{
${ }^{1}$ As sementes da variedade caribaea e as da variedade hondurensis procederam respectivamente de Cuba e da Guatemala. Informação do Dr. Fernando Ferraz, proprietário da Pinusplan Reflorestadora Ltda.
} 
COMISSÃO DE FERTILIDADE DO SOLO DO ESTADO DE MINAS GERAIS - CFSEMG.

Recomendações para o uso de corretivos e fertilizantes em Minas Gerais. $5^{\text {a }}$ aproximação. Viçosa-MG:Universidade Federal de Viçosa, 1999. 359 p.

CHIJIOKE, E.O. Impact on soils of plant growing species in tropic. Rome: FAO, 1980. $121 \mathrm{p}$.

CORRÊA, G. F. Les microrelief "murundus" et leur environnement pédologique dans l'ouest du Minas Gerais, région du plateau central brésilien. 1989. 144f. Tese (Doutorado em Solos) - Université de Nancy I, Vandoeuvre-lesNancy, 1989.

\section{EMPRESA BRASILEIRA DE PESQUISA} AGROPECUÁRIA - EMBRAPA. Centro Nacional de Pesquisa de Solos. Sistema brasileiro de classificação de solos. Rio de Janeiro: 1999. $412 \mathrm{p}$.

LA TORRACA, S.M.; HAAG, H.P.; MIGLIORINI, A.J. Recrutamento e exportação de nutrientes por Pinus elliottii var. elliottii em um latossolo vermelho-escuro na região de Agudos, SP. IPEF, v. 27 , p. 41-47, 1984

LEPSCH, I.F. Influência do cultivo de Eucalyptus e Pinus nas propriedades químicas de solos sob cerrado. Revista Brasileira de Ciência do Solo, n. 4, p. 103-107, 1980.

LILIENFEIN, J. et al. Soil acidification in Pinus caribaea forests on Brazilian savanna Oxisols. Forest Ecology and Management, v. 128, p. $145-157,2000$.

LOPES, M.I.M.S. Influência do cultivo de Pinus sobre algumas características de um Latossolo Vermelho-Escuro primitivamente sob vegetação de cerrado. $1983.90 \mathrm{f}$. Dissertação (Mestrado em Agronomia) - Escola Superior de Agricultura “Luiz de Queiroz", Piracicaba, 1983.

R. Árvore, Viçosa-MG, v.29, n.5, p.691-700, 2005
MALAVOLTA, E. Nutrição mineral e adubação de plantas cultivadas. São Paulo: Pioneira, 1974. 752 p.

MORAIS, F.A. Perfil de distribuição de nutrientes em diferentes sistemas de uso e manejo do solo na região de Iraí de Minas, MG. 1999. 49 f. Monografia (Graduação em Agronomia) - Universidade Federal de Uberlândia, Uberlândia, 2001.

OLIVEIRA, J.B. Pedologia aplicada. Jaboticabal: FUNEP, 2001. 414 p.: il.

SILVA, F.C. (Org.) Manual de análises químicas de solos, plantas e fertilizantes. Brasília: EMBRAPA, 1999. 370 p.

SILVA, L.M.B. Caracterização química de atributos do solo sob diferentes sistemas de uso e manejo estabilizados. 2001. 42f. Monografia (Graduação em Agronomia) - Universidade Federal de Uberlândia, Uberlândia, 2001.

SOUZA, D.M.P.; SOUZA, M.L.P. Alterações provocadas pelo florestamento de Pinus sp. na fertilidade de solos da região de Lapa-PR.

Revista Floresta, v. 12, n. 2, p. 36-50, 1981.

van GOOR, C.L. A nutrição de alguns pinheiros tropicais. Silvicultura em São Paulo, n. 4, p. 313-340, 1966.

VETTORAZZO, S.C.; COUTO, H.T.Z.

Padronização de amostragem para diagnose nutricional e variações nas concentrações de nutrientes nas acículas de Pinus taeda L. I. Macronutrientes. Revista Brasileira de Ciência do Solo, v. 21, p. 51-58, 1997.

ZINN, Y.L. Caracterização de propriedades físicas, químicas e da matéria orgânica de solos nos cerrados sob plantações de Eucalyptus e Pinus. 1998. 85f. Dissertação (Mestrado em Agronomia) - Universidade de Brasília, Brasília, 1998. 